\title{
Development of quantitative metabolomics for Pichia pastoris
}

\author{
Marc Carnicer - André B. Canelas • Angela ten Pierick • \\ Zhen Zeng $\cdot$ Jan van Dam $\cdot$ Joan Albiol $\cdot$ \\ Pau Ferrer · Joseph J. Heijnen $\cdot$ Walter van Gulik
}

Received: 21 January 2011/Accepted: 5 April 2011/Published online: 21 April 2011

(C) The Author(s) 2011. This article is published with open access at Springerlink.com

\begin{abstract}
Accurate, reliable and reproducible measurement of intracellular metabolite levels has become important for metabolic studies of microbial cell factories. A first critical step for metabolomic studies is the establishment of an adequate quenching and washing protocol, which ensures effective arrest of all metabolic activity and removal of extracellular metabolites, without causing leakage of metabolites from the cells. Five different procedures based on cold methanol quenching and cell separation by filtration were tested for metabolomics of Pichia pastoris regarding methanol content and temperature of the quenching solution as key parameters. Quantitative evaluation of these protocols was carried out through mass balance analysis, based on metabolite measurements in all sample fractions, those are whole broth, quenched and washed cells, culture filtrate and quenching and washing solution. Finally, the optimal method was used to study the time profiles of free amino acid and central carbon metabolism intermediates in glucose-limited chemostat cultures. Acceptable recoveries ( $>90 \%$ ) were obtained for all quenching procedures tested. However, quenching at
\end{abstract}

Electronic supplementary material The online version of this article (doi:10.1007/s11306-011-0308-1) contains supplementary material, which is available to authorized users.

M. Carnicer · J. Albiol $(\bowtie) \cdot$ P. Ferrer

Department of Chemical Engineering, Universitat Autònoma de

Barcelona, 08193 Bellaterra (Cerdanyola del Vallès), Spain

e-mail: joan.albiol@uab.cat

A. B. Canelas · A. ten Pierick - Z. Zeng · J. van Dam ·

J. J. Heijnen · W. van Gulik $(\bowtie)$

Department of Biotechnology, Kluyver Centre for Genomics

of Industrial Fermentation, Delft University of Technology,

Julianalaan 67, 2628 BC Delft, The Netherlands

e-mail:w.m.vangulik@tudelft.nl $-27^{\circ} \mathrm{C}$ in $60 \% \mathrm{v} / \mathrm{v}$ methanol performed slightly better in terms of leakage minimization. We could demonstrate that five residence times under glucose limitation are enough to reach stable intracellular metabolite pools. Moreover, when comparing $P$. pastoris and $S$. cerevisiae metabolomes, under the same cultivation conditions, similar metabolite fingerprints were found in both yeasts, except for the lower glycolysis, where the levels of these metabolites in $P$. pastoris suggested an enzymatic capacity limitation in that part of the metabolism.

Keywords Pichia pastoris - Metabolite quantification . Quenching · Chemostat

$\begin{array}{ll}\text { Abbreviations } \\ \text { gDCW } & \text { Grams of dry cell weight } \\ \text { MeOH } & \text { Methanol } \\ \text { G6P } & \text { Glucose-6-phosphate } \\ \text { T6P } & \text { Trehalose-6-phosphate } \\ \text { FBP } & \text { Fructose-1,6-bisphosphate } \\ \text { 6PG } & \text { 6-Phospho gluconate } \\ \text { F6P } & \text { Fructose-6-phosphate } \\ \text { G1P } & \text { Glucose-1-phosphate } \\ \text { M6P } & \text { Mannose-6-phosphate } \\ \text { 2PG } & \text { 2-Phospho glycerate } \\ \text { 3PG } & \text { 3-Phospho glycerate } \\ \text { PEP } & \text { Phosphoenolpyruvate } \\ \text { PYR } & \text { Pyruvate } \\ \alpha \text { KG } & \alpha \text {-Ketoglutarate } \\ \text { G3P } & \text { Glycerol-3-phosphate } \\ \text { S7P } & \text { Sedoheptulose-7-phosphate } \\ \text { UDP-glc } & \text { UDP-glucose } \\ \text { PGI } & \text { Phosphoglucose isomerase } \\ \text { PGM } & \text { Phosphoglucomutase } \\ \text { PMI } & \text { Phosphomannose isomerase }\end{array}$


ENO Enolase

FMH Fumarase

\section{Introduction}

The study of the properties of metabolic networks and their regulation in vivo is a key field in systems biology and, together with proteomic, transcriptomic or fluxomic studies has become a tool towards strain optimization (van Gulik 2010; Bolten et al. 2007; Oldiges et al. 2007; Douma et al. 2010b). Besides, among the main '-omics' technologies, metabolomics is expected to play a significant role in bridging the phenotype-genotype gap, since it amplifies changes in the proteome and provides a better representation of the phenotype of an organism than other methods (Cascante and Marin 2008).

The metabolomics field has experienced a strong development over the recent past, mainly due to the improvements in MS-based analytical procedures (Mashego et al. 2004; van Dam et al. 2002; Dettmer et al. 2007; Oldiges et al. 2007). In combination with the advances in analysis of microbial metabolic fluxes via ${ }^{13} \mathrm{C}$ isotopic labelling (Tang et al. 2009) this has greatly expanded the possibilities for quantitative analysis of metabolic pathways.

Although there have been many improvements in the field, an important step in the study of metabolic reaction networks is to obtain representative and accurate snapshots of the metabolome. Many metabolites, not only those related to the central carbon metabolism but e.g. also free amino acids, have turnover times in the order of seconds or less, which highlight the need for a rapid sampling technique to quantify their actual levels, as well as a proper, leakage-free, quenching procedure to ensure absence of losses or (inter)conversion of metabolites (Canelas et al. 2008; van Gulik 2010). Moreover, if substantial amounts of metabolites are present in the extracellular medium, those need to be efficiently removed. Therefore, a sample treatment procedure allowing separating the intracellular and extracellular metabolite pools, as well as a degradation-free extraction method are required (Canelas et al. 2009).

Although many efforts have been directed towards the development of a universal method, no consensus solution has been found because of the vast diversity in cell properties (Villas-Bôas et al. 2005; Bolten et al. 2007; van Gulik 2010). Recently, proper sampling, quenching, separation and extraction protocols for the intracellular metabolite quantification in Saccharomyces cerevisiae have been presented and successfully applied, allowing accurate, reliable and reproducible metabolite determinations (Canelas et al.
2008, 2009). Unfortunately, it appears that for different microbial species, different sampling and quenching methodologies need to be developed and quantitatively evaluated and validated (Bolten et al. 2007; Bolten and Wittmann 2008; Taymaz-Nikerel et al. 2009).

The methyltrophic yeast Pichia pastoris has become an outstanding cell factory, not only for recombinant protein production (Cereghino et al. 2002; Macauley-Patrick et al. 2005; Cos et al. 2006; Bollok et al. 2009) but also for producing other active pharmaceutical ingredients (Pscheidt and Glieder 2008). Notably, some metabolic network studies in $P$. pastoris, focused on the metabolic effects of recombinant protein production have been reported. However, these were performed using stable isotope labeling $\left({ }^{13} \mathrm{C}\right)$ whereby only isotopologue ratios, but no absolute metabolite level determinations were needed (Solà et al. 2004, 2007; Heyland et al. 2010; Baumann et al. 2010). Moreover, two genome-scale metabolic network models have been recently published opening the door to a better understanding of the metabolic network of P. pastoris (Sohn et al. 2010; Chung et al. 2010).

Recently, the impact of the oxygen availability on biomass composition (Carnicer et al. 2009), as well as on the transcriptome, proteome and metabolic fluxes (Baumann et al. 2008) of recombinant $P$. pastoris grown in glucose limited chemostat cultures has been investigated. In the present study, the same strain and similar conditions were applied as experimental model for further metabolomic studies. In particular, a systematic evaluation of five quenching protocols applied to $P$. pastoris was carried out in order to obtain a reliable intracellular metabolite quantification method. Methanol concentration and temperature were the selected variables to be optimized for minimizing metabolite leakage during quenching and subsequent washing. Thereafter, the best performing quenching protocol was used in two applications: (1) determination of the time needed for each intracellular metabolite to reach a metabolite steady state and (2) a metabolome comparison between $P$. pastoris and $S$. cerevisiae.

\section{Materials and methods}

\subsection{Strain and cultivation conditions}

Analytical grade reagents were supplied by Sigma-Aldrich. HPLC-grade methanol and ethanol were supplied by J.T. Baker.

In this study the $P$. pastoris $\mathrm{X}-33$ (wild type phenotype, Invitrogen) transformed with $\mathrm{pGAP} \alpha \mathrm{A}$ (Invitrogen) as mock vector was used. Chemostat cultivation was performed in a 7-1 fermentor (Applikon, The Netherlands) with a working volume of 41 . The culture media used were 
derived from previously described media (Baumann et al. 2008). In particular, the $\mathrm{C}$-source of the chemostat medium was reduced to obtain a steady state biomass concentration of approximately $4.5 \mathrm{~g} / \mathrm{l}$. The other components were adjusted accordingly to obtain similar residual concentrations as with the original medium, thereby considering the biomass composition of P. pastoris (Carnicer et al. 2009). The composition of the batch medium was: $8 \mathrm{~g} / \mathrm{l}$ glycerol, $0.9 \mathrm{~g} / \mathrm{l}$ citric acid monohydrate, $12.6 \mathrm{~g} / \mathrm{l}\left(\mathrm{NH}_{4}\right)_{2} \mathrm{HPO}_{4}$, $0.5 \mathrm{~g} / 1 \quad \mathrm{MgSO}_{4} \cdot 7 \mathrm{H}_{2} 0, \quad 1.5 \mathrm{~g} / 1 \quad \mathrm{KH}_{2} \mathrm{PO}_{4}, \quad 0.02 \mathrm{~g} / 1$ $\mathrm{CaCl}_{2} \cdot 2 \mathrm{H}_{2} \mathrm{O}, 5 \mathrm{ml} / \mathrm{l}$ trace salt solution, $2 \mathrm{ml} / \mathrm{l}$ Biotin solution $(0.2 \mathrm{~g} / \mathrm{l})$. The composition of the chemostat medium was: $8.80 \mathrm{~g} / 1$ glucose monohydrate, $0.92 \mathrm{~g} / 1$ citric acid monohydrate, $2 \mathrm{~g} / \mathrm{l}\left(\mathrm{NH}_{4}\right)_{2} \mathrm{HPO}_{4}, 0.3 \mathrm{~g} / \mathrm{l} \mathrm{MgSO}_{4} \cdot 7 \mathrm{H}_{2} 0$, $1.4 \mathrm{~g} / 1 \mathrm{KH}_{2} \mathrm{PO} 4,0.01 \mathrm{~g} / 1 \mathrm{CaCl}_{2} \cdot 2 \mathrm{H}_{2} 0,0.5 \mathrm{ml} / \mathrm{l}$ trace salt solution, $0.3 \mathrm{ml} / 1$ Biotin $(0.2 \mathrm{~g} / \mathrm{l})$. The trace salts solution was the same as described previously (Baumann et al. 2008)

One liter shake flask containing $300 \mathrm{ml}$ of YPD medium (10 g/l yeast extract, $20 \mathrm{~g} / 1$ peptone, $10 \mathrm{~g} / 1$ glucose) was inoculated with a $1.0 \mathrm{ml}$ cryostock of $P$. pastoris cells. The culture was grown for approximately $24 \mathrm{~h}$ at $30^{\circ} \mathrm{C}$ with shaking at $200 \mathrm{rpm}$, and used to inoculate the reactor. After complete termination of the batch phase, approximately $24 \mathrm{~h}$ after performing the inoculation, the feed of the chemostat culture was started. During the chemostat, the cells were grown under carbon-limited conditions at a dilution rate (D) of $0.1 \mathrm{~h}^{-1}$, and an aeration rate of $0.5 \mathrm{vvm}$, controlled by mass flow meters (5850 Smart Mass Flow Controller, Brooks Instrument). During the entire cultivation the $\mathrm{pO}_{2}$ was maintained above $50 \%$ ensuring fully aerobic conditions. The $\mathrm{O}_{2}$ and $\mathrm{CO}_{2}$ concentrations in the bioreactor off-gas were measured on-line using a combined paramagnetic/infrared analyzer (NGA 2000, Rosemount, USA). Pressure, $\mathrm{pH}$, stirring speed and temperature were maintained at 1.2 bars, $\mathrm{pH} 5$ (with $20 \%$ $\left.\mathrm{v} / \mathrm{vNH}_{3}\right), 400 \mathrm{rpm}$ and $25^{\circ} \mathrm{C}$, respectively.

In the leakage evaluation experiment, a first chemostat culture was maintained for five residence times until a stable off-gas reading was obtained. This measure was used as an indicator for obtaining a steady-state condition. During steady state five duplicate samples were taken, i.e. two for each quenching protocol, for metabolite measurements in quenched and washed cells, and in quenching and washing solutions. Furthermore three filtrate samples and five whole broth samples were taken.

Once the protocols were evaluated, using the best of the tested protocols, two additional chemostat cultures were run to study the time needed for $P$. pastoris to reach a metabolic pseudo steady-state. Duplicate samples for intracellular metabolite measurement were taken approximately each $24 \mathrm{~h}$ during a period of 10 residence times.

\subsection{Sampling}

Samples for intracellular metabolite analysis were taken using a dedicated rapid-sampling setup (Lange et al. 2001). With this setup approximately, $0.63 \pm 0.01 \mathrm{~g}$ of broth was rapidly withdrawn and immediately injected in $5 \mathrm{ml}$ of precooled quenching solution. The tubes were quickly mixed by vortexing and introduced in the filtration unit after weighting the tube (Douma et al. 2010a). All sampling tubes were weighted before and after the sampling procedure in order to determine the exact amount of sample taken. Briefly, the cell suspensions were filtered with membrane disk filters (Pall Corporation, East Hills, NY, USA, $47 \mathrm{~mm}$ diameter, $0.45 \mu \mathrm{m}$ pore size) using a vacuum pump. A washing step was performed to remove as much extracellular metabolites as possible. This washing step consisted of rapidly pouring a fresh amount of $10 \mathrm{ml}$ of the previously mentioned quenching solution on the filter cake as soon as the biomass started to fell dry, thereby maintaining the vacuum on the filtration unit. The average total contact time between the cells and the quenching solution (from taking the sample until the washing solution completely passed through the filter) was $1 \mathrm{~min} \pm 7 \mathrm{~s}$.

For accurate quantification purposes by using Isotope Dilution Mass Spectrometry (Mashego et al. 2004; Wu et al. 2005), $120 \mu \mathrm{l}$ of a ${ }^{13} \mathrm{C}$ internal standard solution $\left(0^{\circ} \mathrm{C}\right)$ was pipetted on top of the dry filter cake. The ${ }^{13} \mathrm{C}$ internal standard solution contained all relevant metabolites as $\mathrm{U}-^{13} \mathrm{C}$-labeled isotopes and was obtained from a $S$. cerevisiae fed-batch culture grown on $100 \% \mathrm{U}^{13}{ }^{13} \mathrm{C}$-labeled glucose and ethanol. Metabolite extraction with $75 \%(\mathrm{v} / \mathrm{v})$ aqueous ethanol at $95^{\circ} \mathrm{C}$ and further sample processing were carried out as described previously (Douma et al. 2010a).

The quenching and washing liquids (QWS) were collected in $50 \mathrm{ml}$ Falcon tubes. After thorough mixing, aliquots of $3.0 \mathrm{ml}$ of the solutions were mixed with $120 \mu \mathrm{l}$ of a ${ }^{13} \mathrm{C}$ internal standard solution and subsequently extracted in $75 \%(\mathrm{v} / \mathrm{v})$ aqueous ethanol at $95^{\circ} \mathrm{C}$, to eliminate possible enzymatic activity in these samples. The exact sample amounts taken were determined by weighting all tubes before and after sampling.

Samples from the culture filtrate $(\mathrm{CF})$ and the complete culture broth (WB) were withdrawn and further processed as described earlier (Canelas et al. 2008).

\subsection{Metabolite analysis}

Metabolite quantification was carried out with LC-ESIMS/MS and GC-MS based isotope dilution mass spectrometry (IDMS) (Canelas et al. 2009). Each sample was analyzed in duplicate. In total, 37 metabolites, with a wide variety of chemical and physical properties, were analyzed. 
Table 1 Tested quenching protocols to investigate the effects of temperature and methanol concentration on metabolite leakage

\begin{tabular}{llll}
\hline Protocol & $\begin{array}{l}\text { Temperature } \\
\left({ }^{\circ} \mathrm{C}\right)\end{array}$ & $\begin{array}{l}\text { Concentration of } \\
\text { methanol solutions } \\
(\mathrm{v} / \mathrm{v})(\%)\end{array}$ & $\begin{array}{l}\text { Concentration of } \\
\text { methanol after } \\
\text { sampling }(\mathrm{v} / \mathrm{v})(\%)\end{array}$ \\
\hline A & -27 & 40 & 36 \\
B & -27 & 60 & 54 \\
C & -40 & 60 & 54 \\
D & -40 & 80 & 71 \\
E & -40 & 100 & 89 \\
\hline
\end{tabular}

However, only 34 of these could actually be measured, because the concentrations of glyoxylate, mannitol-6phosphate and fructose-2,6-bisphosphate were found to be below the detection limit in all the samples analyzed.

\subsection{Consistency check and data reconciliation}

The amount of each metabolite was quantified in different sample fractions, that is, in whole broth (WB), quenched/ washed cells (QC), culture filtrate (CF) and quenching + washing liquid (QWS). Because the ${ }^{13} \mathrm{C}$ internal standard mix was in all cases added to the different sample fractions prior the metabolite extraction procedure, possible metabolite losses due to partial degradation could be corrected for. This implies that the following mass balance should be satisfied for each metabolite $i$ :
$M_{i}(\mathrm{WB})=M_{i}(\mathrm{CF})+M_{i}(\mathrm{QC})+M_{i}($ leakage $)$

This mass balance allows calculating, from measurements in total broth, filtrate and cells, which amount was lost into the quenching and washing solutions as a result of leakage. However, because in addition also metabolite quantifications were carried out in the quenching + washing solutions, a second mass balance can be written, namely:

$M_{i}(\mathrm{WB})=M_{i}(\mathrm{QC})+M_{i}(\mathrm{QWS})$

This balance states that the total amount of each metabolite (i.e. the extracellular + intracellular amount) present in a whole broth sample should be equal to the amounts measured in the cell cake sample and in the quenching + washing solution. This means that the data set contains redundant information, i.e. more information than strictly necessary to quantify metabolite leakage, allowing to perform a statistical consistency check, using the calculated $\chi^{2}$-distributed consistency index $h$, as well as data reconciliation (van der Heijden et al. 1994).

Briefly the data reconciliation was performed as follows: under the constraint that the mass balances (Eqs. 1, 2) should be satisfied, the best estimates of the measured metabolite amounts in the different sample fractions as well as the calculated amount of leakage were obtained by least squares minimization of the differences between the measured and estimated amounts, weighed by their measurement errors (Verheijen 2010).
Fig. 1 Full mass balances of some representative metabolites. The letters below the bars represent the five different treatments tested. $A-27^{\circ} \mathrm{C}, 40 \% \mathrm{MeOH}$; $B-27^{\circ} \mathrm{C}, 60 \% \mathrm{MeOH}$; $C-40^{\circ} \mathrm{C}, 60 \% \mathrm{MeOH}$; $D-40^{\circ} \mathrm{C}, 80 \% \mathrm{MeOH}$ and $E-100^{\circ} \mathrm{C}, 100 \% \mathrm{MeOH}$
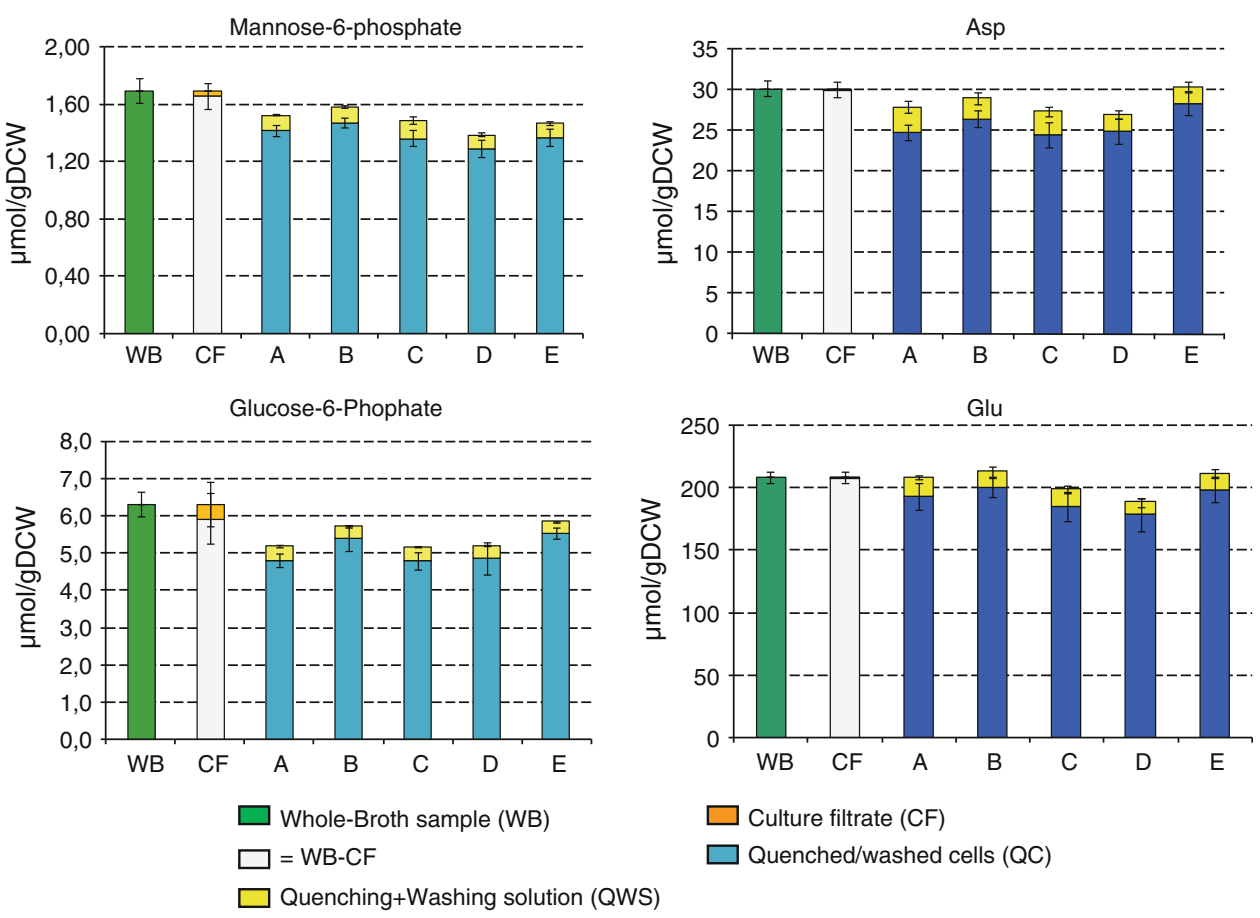
Table 2 Direct and differential intracellular quantification comparison

\begin{tabular}{|c|c|c|c|c|}
\hline \multirow[t]{2}{*}{$\mu \mathrm{mol} / \mathrm{gDCW}$} & \multicolumn{2}{|c|}{ ICS protocol B } & \multicolumn{2}{|c|}{$\mathrm{WB}-\mathrm{CF}$} \\
\hline & Value & SD & Value & SD \\
\hline G6P & 5.4 & 0.3 & 5.9 & 0.7 \\
\hline F6P & 1.22 & 0.09 & 1.21 & 0.06 \\
\hline FBP & 0.70 & 0.07 & b & b \\
\hline 2-3PG & 1.93 & 0.22 & 2.14 & 0.12 \\
\hline PEP & 0.33 & 0.04 & 0.33 & 0.02 \\
\hline Citric acid & 7.3 & 0.6 & c & c \\
\hline$\alpha \mathrm{KG}$ & 2.09 & 0.15 & 2.25 & 0.18 \\
\hline Succinate & 2.94 & 0.18 & 2.79 & 0.27 \\
\hline Fumarate & 1.37 & 0.06 & 1.09 & 0.15 \\
\hline Malate & 6.9 & 0.6 & 7.0 & 0.9 \\
\hline G1P & 0.80 & 0.06 & 0.68 & 0.06 \\
\hline $6 \mathrm{PG}^{\mathrm{a}}$ & 0.67 & 0.10 & 0.57 & 0.09 \\
\hline M6P & 1.47 & 0.03 & 1.66 & 0.09 \\
\hline Trehal.-6P & 0.09 & 0.01 & b & b \\
\hline G3P & 0.06 & 0.01 & 0.05 & 0.04 \\
\hline S7P & 2.08 & 0.09 & 2.08 & 0.16 \\
\hline UDP-glc & 1.18 & 0.31 & 0.95 & 0.17 \\
\hline Ala & 23.5 & 0.1 & 23.8 & 2.4 \\
\hline Val & 1.97 & 0.07 & 1.99 & 0.05 \\
\hline Leu & 1.48 & 0.10 & 1.50 & 0.02 \\
\hline Ile & 0.53 & 0.04 & 0.55 & 0.01 \\
\hline $\mathrm{Thr}^{\mathrm{a}}$ & 3.94 & 0.02 & 4.19 & 0.12 \\
\hline Pro & 10.8 & 0.4 & 11.7 & 0.2 \\
\hline Asn & 7.1 & 0.2 & 7.6 & 0.2 \\
\hline Asp & 26.4 & 1.0 & 30.1 & 0.9 \\
\hline Met & 0.97 & 0.15 & 1.03 & 0.15 \\
\hline Glu & 200 & 8 & 208 & 5 \\
\hline Phe & 0.51 & 0.04 & 0.52 & 0.01 \\
\hline $\operatorname{Gln}^{\mathrm{a}}$ & 177 & 8 & 178 & 5 \\
\hline Orn & 56 & 3 & 54.8 & 0.6 \\
\hline Lys & 12.8 & 0.7 & 12.9 & 0.4 \\
\hline His & 7.4 & 0.5 & 7.3 & 0.1 \\
\hline Tyr & 0.80 & 0.06 & 0.82 & 0.02 \\
\hline Trp & 0.24 & 0.02 & 0.23 & 0.01 \\
\hline
\end{tabular}

${ }^{a}$ No $\mathrm{CF}$ quantification available. WB-CF was replaced by WB measurements

${ }^{\mathrm{b}}$ Metabolite lower than the detection limit

c Metabolite higher than the calibration range

SD Standard deviation

\section{Results and discussion}

\subsection{Chemostat cultivations}

Pichia pastoris was grown in aerobic, glucose limited chemostat cultures at a dilution rate of $0.1 \mathrm{~h}^{-1}$. Under these conditions biomass and carbon dioxide were the only products. Once steady state was obtained, the consumption rates of glucose and oxygen and the production rates of biomass and carbon dioxide were calculated from measurements of biomass dry weight, residual glucose and the concentrations of oxygen and carbon dioxide in the off-gas.

The experimental data consistency was verified using standard data reconciliation procedures, under the constraint that the elemental conservation relations were satisfied (van der Heijden et al. 1994; Verheijen 2010). For all chemostat cultivations performed the statistical consistency test, carried out with a confidence level of $95 \%$, was acceptable, indicating that there was no proof for gross measurement errors. Therefore, under the applied chemostat conditions, the balanced steady state input-output rates obtained were $-0.97( \pm 0.01), 3.65( \pm 0.01), 2.19( \pm 0.09)$ and $-2.11( \pm 0.09) \mathrm{mmol} /(\mathrm{gDCW} \cdot \mathrm{h})$ for glucose uptake rate, Biomass production, $\mathrm{CO}_{2}$ evolution rate and oxygen uptake rate respectively. As expected, the rates were equivalent to the ones obtained in previous studies, thereby making the data sets comparable (Carnicer et al. 2009; Baumann et al. 2010).

\subsection{Quenching optimization}

\subsubsection{Effect of methanol content and quenching temperature}

To study the effect of the quenching procedure on metabolite leakage, a full mass balance analysis was performed as described in Canelas et al. (2008). Briefly, the fate of the metabolites was identified by quantification of metabolite levels in four different fractions: whole-broth fraction (WB), culture filtrate (CF), quenched/washed cells (QC) and quenching + washing methanol solution (QWS). The actual intracellular metabolite levels were estimated from the difference between the levels measured in whole broth (WB) and culture filtrate (CF) (Canelas et al. 2008; Taymaz-Nikerel et al. 2009). However, this approach for estimation of intracellular metabolite levels has drawbacks compared to the direct measurement of quenched/washed cells. In particular, it requires a double analytical effort, as two samples have to be analyzed, and whole broth and supernatant samples may contain high amounts of salts, potentially interfering (ion suppression) with the analytical techniques (Canelas et al. 2008). On the other hand, in the quenched/washed cells, metabolism needs to be properly arrested, while avoiding leakage and degradation.

Therefore, a comparison of the determined intracellular metabolite levels between the direct measurement (QC) and the differential method (WB-CF) was performed for the five different variations of the cold methanol quenching protocols, to determine for which condition metabolite leakage from $P$. pastoris cells was minimal. The methanol 

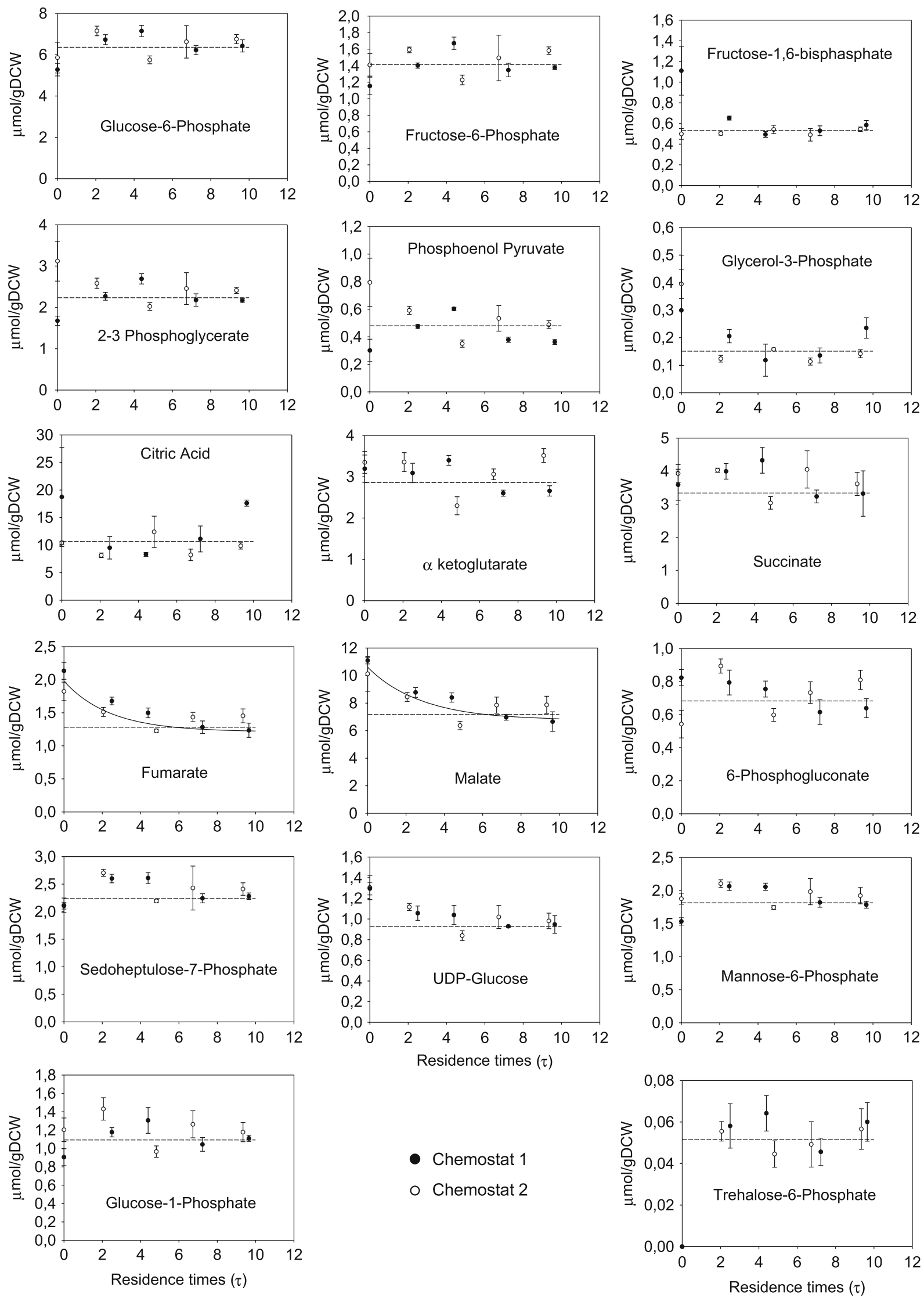
4Fig. 2 Evolutionary changes in intracellular central carbon metabolite pools associated with adaptation to glucose-limited conditions in two chemostats. The lines represent the exponential decay curve fitted and dashed lines the weighted average of the last five residence times

content of the quenching solution and the quenching temperature were the parameters changed to investigate their impact on possible metabolite leakage as shown in Table 1.

The metabolites analyzed consisted of a wide range of different chemical compounds, such as phosphorylated intermediates, organic acids and amino acids. In some cases the fate of the metabolite could not be followed properly and therefore the mass balances could not be calculated. For example, the amounts of FBP and T6P in CF, QWS and WB were below the detection limits while for citric acid the differential method could not be applied due to a too high extracellular level outside measuring range and therefore, QC was the only measurement which could be carried out.

As a first step of the data evaluation, the average mass balance closure was calculated ((QC + QWS)/WB) being on average $108 \%( \pm 24 \%)$. This was considered acceptable due to the analytical challenge and was in the range of values obtained in previous studies employing the mass balance approach (Canelas et al. 2008). Nevertheless, the data consistency was also checked individually for each metabolite to detect any gross errors in the measurements (see Sect. 3.2.2).

Secondly, the full mass balances were analyzed metabolite by metabolite. In Fig. 1 some examples are shown, representing different classes of metabolites. Comparison of the intracellular metabolite levels obtained with the differential method (WB-CF) and the quenched cell measurements (QC) revealed that metabolite leakage occurred to some extent in all treatments, independent of the methanol content of the quenching solution, the quenching temperature or the compound type. This could be attributed to the so called "cold shock" phenomenon, leading to a sudden release of metabolites from the cells when the broth is rapidly cooled, as reported for the bacterium Corynebacterium glutamicum (Wittmann et al. 2004; Wellerdiek et al. 2009).

However, in a previous study performed with the yeast S. cerevisiae (Canelas et al. 2008) the extent of metabolite leakage was found to be heavily influenced by the methanol content used in the quenching solution; whereby decreasing the methanol content resulted in increased leakage. These results highlight the need to fine tune the quenching protocol for each microorganism. On the other hand, the much smaller influence of the methanol content observed for $P$. pastoris could also be attributed to the shorter contact time with the quenching solution (about $1 \mathrm{~min}$ ) when applying the cold filtration method. In the work of (Canelas et al. 2008) the cold centrifugation method was used, whereby the cells were between 20-30 min in contact with the quenching solution. Detailed data of the metabolite quantification results for each protocol is provided as supplementary material (see Additional file 1).

\subsubsection{Protocol evaluation}

From the evaluation of the mass balances for each metabolite, as shown in Fig. 1, no clear distinction could be made between the different protocols. Therefore, data reconciliation was applied under the constraint that for each metabolite both mass balances which could be evaluated (Eqs. 1, 2) were satisfied (see Sect. 2). Thus for each metabolite the data consistency was evaluated at $95 \%$ confidence level and one degree of freedom. Under these conditions the calculated, $\chi^{2}$-distributed, consistency index $h$ should have a value of 3.84 or less. The calculated $h$-index values are shown in the Appendix. When inspecting these values it can be seen that in some cases proof is obtained for gross measurements errors $(h>3.84)$. Only, for two metabolites, namely Pyr and Gly, the data were inconsistent for all the treatments tested. In case of Pyr these systematic errors could be related to quantification errors in Pyr peaks due to analytical difficulties. However, for Gly no clear reason could be identified for the data inconsistency.

Interestingly, the free amino acid quantifications showed higher global consistency, having lower $h$ values compared to the central carbon metabolites, thereby indicating more accuracy in the GC-MS analysis of amino acids.

In the subsequent calculation of the average metabolite yield $(\mathrm{QC} /(\mathrm{WB}-\mathrm{CF})$ ) for the different quenching protocols (A to E) only the statistically consistent data $(h<3.84)$ were taken into account. The recoveries and the standard error for each protocol were 93.1 \pm 1.1 , $95.4 \pm 0.7,92.6 \pm 1.3,94.3 \pm 1.1$ and $93.4 \pm 1.5$ for Protocols A to E respectively (Table 1). Although the differences were small, application of Protocol B resulted in the highest average yield, being approximately $95 \%$.

Consequently, based on the data consistency and average metabolite yield, protocol $\mathrm{B}$ was considered as the optimum quenching procedure for quantification of the intracellular metabolites in P. pastoris. Moreover, in order to evaluate the applicability of direct measurement using quenching protocol $\mathrm{B}$, the obtained results were compared with the results obtained using with the differential method (see in Table 2). To test for significant differences between the results obtained with both methods a two tailed Student's T test was performed. Results show that, only in the case of Asp the obtained values were significantly 

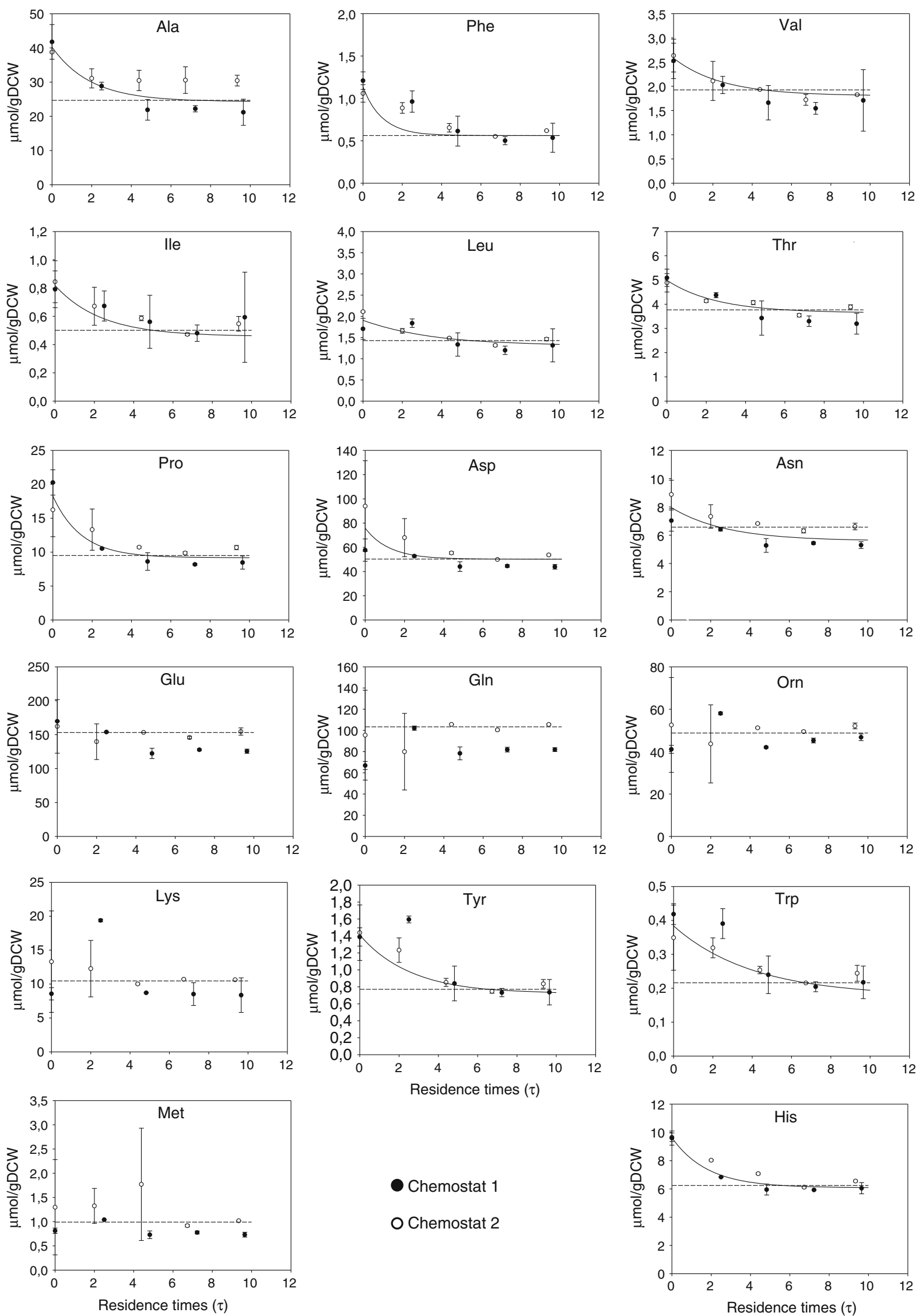
4Fig. 3 Evolutionary changes in intracellular amino acid pools associated with adaptation to glucose-limited conditions in two chemostats. The lines represent the exponential decay curve fitted and dashed lines the weighted average of the last five residence times. *Data taken from Canelas et al. $(2008,2011)$

different, while no significant difference was detected in any other metabolite.

Therefore it is concluded that, in spite of the fact that for one metabolite (Asp) the values were slightly deviated, direct measurement, using quenching protocol $\mathrm{B}$ is the preferred methodology for quantification of intracellular metabolites in $P$. pastoris. In comparison to the differential method, this procedure results, on average, in smaller measurement errors. Furthermore, it requires less analytical effort because for each measurement only one sample has to be analyzed instead of two (WB and CF) for the differential method. Besides, as for the $\mathrm{WB}$ and $\mathrm{CF}$ no medium components are eliminated, they could potentially interfere (ion suppression) with the analytical techniques (Canelas et al. 2008). For these reasons, we applied, for the experiments described in the next section, quenching protocol $\mathrm{B}$ for the quantification of the $P$. pastoris metabolome.

In a recent paper of Tredwell et al. (2011), published after our paper had been submitted, also the evaluation of procedures for sampling and cold methanol quenching of $P$. pastoris for metabolome analysis is described. Although the quenching conditions were different from ours with respect to temperature, methanol content and the addition of buffers, they also found that the different variations of the cold methanol quenching method used gave very similar results. Apparently $P$. pastoris is a relatively robust microorganism, resistant to cold methanol quenching with respect to metabolite leakage. Also Tredwell et al. present baseline metabolome data for chemostat cultured P. pastoris cells and present a comparison between the P. pastoris and $S$. cerevisiae metabolome. The conditions they applied were, however, highly different with respect to the strains of $P$. pastoris and $S$. cerevisiae, the cultivation conditions (batch vs. chemostat) and the substrates (methanol and glycerol vs. glucose) used. Most important difference is that the study of Tredwell at all aimed at metabolic profiling, using non-targeted analytical techniques, whereas in our work a targeted, quantitative approach was used, applying isotope dilution mass spectrometry (IDMS) aimed at obtaining highly accurate metabolome data.

\subsection{Steady-state evaluation}

Typically, the first approach in metabolomic studies is the quantification of the (pseudo) steady-state metabolite concentrations in order to obtain a better understanding of the cell behaviour during fixed environmental conditions.

For measurement of metabolite levels in steady state chemostat cultivation, it is generally assumed, but seldom verified, that the intracellular metabolites have reached their steady state levels after five residence times. To verify this for chemostat cultivation of our $P$. pastoris strain, two replicate chemostat cultures were carried out. Rapid sampling, combined with quenching according to protocol $\mathrm{B}$ and filtration, was applied for intracellular metabolite measurement at $24 \mathrm{~h}$ intervals, from the start of the chemostat phase until a period of 10 residence times.

For the metabolites of which the levels evolved towards different steady state values, an exponential decay curve was fitted (Eq. 3), whereby each measurement was weighted by its standard deviation.

$C_{i}(t)=C_{i, \mathrm{SS}}+\left(C_{i}(0)-C_{i, \mathrm{SS}}\right) \cdot \mathrm{e}^{-b t}$

Herein $C_{i}(0)$ is the average quantification pool for each metabolite at the end of the batch phase, $C_{i, \mathrm{Ss}}$ is the steady state level and $\mathrm{b}$ is a time constant.

For the metabolites which were already at a stable level from the beginning, a weighted average of the five last residence times was taken as the steady state value. Moreover, this was also done for the metabolites which showed changing levels to compare the results from both calculations.

The results are shown in Figs. 2 and 3. Globally, the small differences between the profiles obtained for the two chemostats shows the high reproducibility of the cultivations. Furthermore the relatively small errors in the individual metabolite measurements indicate a high analytical

Table 3 Physiological parameters of $P$. pastoris and S. cerevisiae grown in aerobic carbon-limited chemostat at a D of $0.1 \mathrm{~h}^{-1}$

\begin{tabular}{llllll}
\hline & $\begin{array}{l}Y_{\mathrm{sx}}{ }^{\mathrm{a}} \\
\mathrm{gDCW} / \mathrm{C}-\mathrm{mol} \mathrm{glc}\end{array}$ & $\begin{array}{l}q_{\mathrm{glc}} \\
\mathrm{mmol} /(\mathrm{gDCW} \cdot \mathrm{h})\end{array}$ & $\begin{array}{l}q_{\mathrm{O}_{2}} \\
\mathrm{mmol} /(\mathrm{gDCW} \cdot \mathrm{h})\end{array}$ & $\begin{array}{l}q_{\mathrm{CO}_{2}} \\
\mathrm{mmol} /(\mathrm{gDCW} \cdot \mathrm{h})\end{array}$ & $\begin{array}{l}\mathrm{RQ} \\
\mathrm{mmol} /(\mathrm{gDCW} \cdot \mathrm{h})\end{array}$ \\
\hline P. pastoris & 16.90 & 0.97 & 2.11 & 2.19 & 1.04 \\
S. cerevisiae $^{\mathrm{c}}$ & 14.80 & 1.10 & 2.70 & 2.87 & 1.06 \\
\hline
\end{tabular}

\footnotetext{
${ }^{a}$ Yield of biomass (gDCW/(C-mol glc consumed)

${ }^{\mathrm{b}}$ Respiratory Coefficient $\left(q_{\mathrm{CO}_{2}} / q_{\mathrm{O}_{2}}\right)$

c Data taken from (Canelas et al. 2008)
} 
Fig. 4 Intracellular metabolite comparison of $P$. pastoris and $S$. cerevisiae. The bubble areas are proportional to the pool sizes of each intracellular metabolite.

The numbers indicate the exact pool size in $\mu \mathrm{mol} / \mathrm{gDCW}$. *Data taken from Canelas et al. (2008)

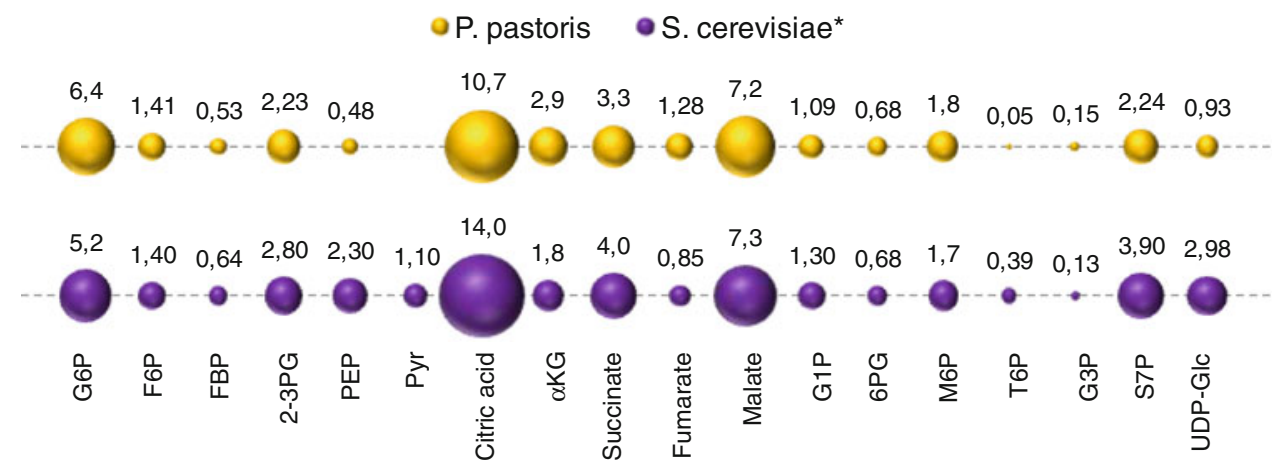

$\begin{array}{llllllllllll}10,7 & 2,9 & 3,3 & 1,28 & 7,2 & 1,09 & 0,68 & 1,8 & 0,05 & 0,15 & 2,24 & 0,93\end{array}$

14,0 reproducibility for most of the metabolites reinforcing the choice of the Protocol B as the optimum one for the intracellular metabolite quantification. However, some biological differences were observed for few metabolites when the pool sizes of the two independent chemostat were compared.

Focusing on the profiles of metabolites from the upper glycolysis and the pentose phosphate pathway (Fig. 2), for most metabolites no significant changes were observed during the 10 residence times of chemostat cultivation. This could be explained by the absence of pool size differences between the two growth conditions (batch phase and continuous phase) or a very fast adaptation after shifting from batch to continuous operation mode for that part of the metabolism. However, a slight increase of the G6P and F6P levels were observed as well as a more pronounced decrease in the pool size of G3P, probably because the carbon source was changed from glycerol to glucose when the continuous culture phase was started. In the TCA cycle, fumarate and malate could be described using an exponential decay profile as indicated by the fitted curve showing slower adaptation compared to other metabolites. Moreover, no changes were observed in the levels of other TCA cycle metabolites, such as $\alpha \mathrm{KG}$ and succinate, during 10 residence times. A metabolite related to the storage metabolism, such as T6P, was below the detection limit at the end of the batch phase and increased to higher steady state levels in less than $24 \mathrm{~h}$, reflecting the regulatory role of this metabolite in the glucose uptake as described elsewhere (Eastmond and Graham 2003). Also, the UDP-glucose level was higher during the batch phase and, after $24 \mathrm{~h}$ of chemostat cultivation, reached a lower stable value showing a different regulation of storage metabolism during the two cultivation conditions.

It is remarkable that most of the measured central metabolites show no or relatively little change during the transition between batch cultivation on glycerol and chemostat cultivation on glucose as sole carbon source. One reason could be that the decrease in the growth rate during the transition is relatively small, i.e. from $\mu=0.17$ to $0.1 \mathrm{~h}^{-1}$.

Recently, a study was performed comprising a total of 32 growth conditions of $S$. cerevisiae, covering a range of growth rates from 0.02 to $0.38 \mathrm{~h}^{-1}$ (Canelas et al. 2011). The complete set of conditions spanned a large range of metabolic fluxes with, as the median for 27 reactions investigated, a 35 fold change. The associated changes in intracellular metabolite levels for these highly different flux profiles were, however, much smaller, with, as the median, a maximum change of 3.5 fold. This shows how tightly metabolism is regulated to keep the metabolite levels between narrow regions (homeostasis).

The evolution of the free amino acid pools are shown in Fig. 3. The observation that the levels of many free amino acids decreased to lower levels could be related to the change in growth rate between the batch phase $\left(\mu=0.17 \mathrm{~h}^{-1}\right)$ and the chemostat phase $\left(\mu=0.1 \mathrm{~h}^{-1}\right)$. This relation is strengthened when considering that usually

Table 4 Mass action ratios of some enzymes related to the central carbon metabolism of S. cerevisiae and P. pastoris

\begin{tabular}{lllll}
\hline Enzyme & Mass action ratios & $\begin{array}{l}\text { This study } \\
\text { P. pastoris }\end{array}$ & $\begin{array}{l}\text { Canelas et al. (2008) } \\
\text { S. cerevisiae }\end{array}$ & $\begin{array}{l}\text { Canelas et al. (2011) } \\
\text { In vivo } k_{\text {eq }}\end{array}$ \\
\hline PGI & F6P/G6P & $0.22 \pm 0.11$ & $0.26 \pm 0.05$ & $0.259 \pm 0.002$ \\
PGM & G1P/G6P & $0.13 \pm 0.09$ & $0.05 \pm 0.02$ & $0.063 \pm 0.004$ \\
PMI & M6P/F6P & $1.27 \pm 0.06$ & $1.17 \pm 0.03$ & $1.183 \pm 0.013$ \\
ENO & PEP/2-3PG & $1.67 \pm 0.10$ & $3.99 \pm 0.13$ & $4.01 \pm 0.09$ \\
FMH & Malate/fumarate & $5.25 \pm 0.38$ & $4.82 \pm 0.12$ & $5.15 \pm 0.14$
\end{tabular}

S. cerevisiae data were derived from Canelas et al. (2008). The apparent in vivo $k_{\mathrm{eq}}$ were taken from Canelas et al. (2011)

${ }^{\text {a }}$ The $2 \mathrm{PG}$ total amounts were calculated assuming that $3 \mathrm{PG}$ and $2 \mathrm{PG}$ were in equilibrium $\left(k_{\mathrm{eq}}=0.1\right)$ 


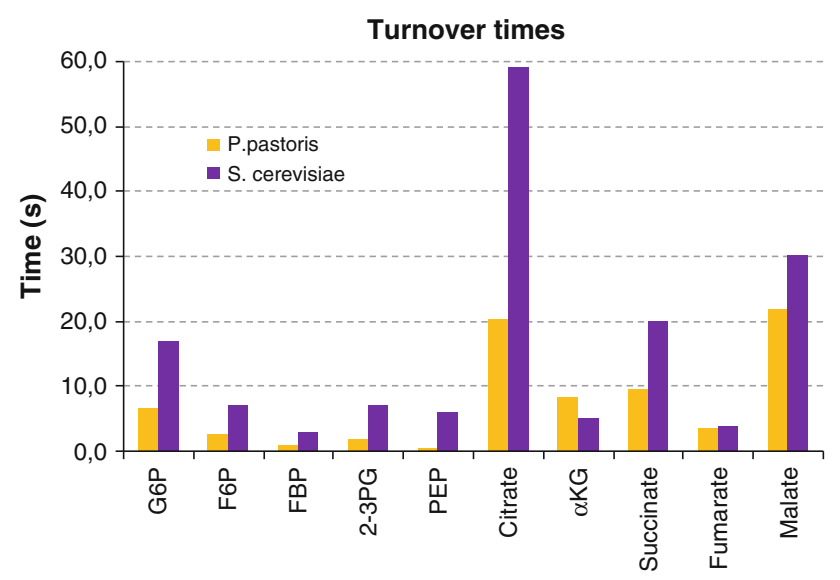

Fig. 5 Intracellular turnover times of the central metabolism metabolites of P. pastoris and S. cerevisiae. *Data taken from Canelas et al. (2008)

the total protein content in the biomass increases with the growth rate, being higher at higher growth rates (Abbott et al. 1974; Nielsen 1997) and therefore requiring higher protein synthesis rates. Moreover, a recent genomic-scale metabolic network reconstruction for P. pastoris showed higher biosynthetic amino acid flux requirements when the cells were grown on glycerol compared to glucose (Chung et al. 2010). Remarkably, the amino acids belonging to the glutamate family, the largest intracellular amino acid pool, did not show any significant changes during 10 residence times of chemostat cultivation, which could also be the result of lack of variation in terms of pool sizes between the two conditions or to a fast rate of adaptation to the new growth conditions. On the other hand, for the other amino acids, the fitted curve could adequately describe the pool trends highlighting that, for most of them, five residence times were sufficient to achieve the steady-state values considering the experimental error. In these amino acids, the steady-state values of the fitted curves $\left(C_{i, \mathrm{Ss}}\right.$ parameter in Eq. 3) were compared to the values from the weighted average of the last five residence times, showing less than $10 \%$ difference between two calculations except for Trp and Asn with a 17 and $15 \%$ of difference, respectively. These results were considered satisfactory considering the analytical challenge involved (See supplementary material 2 for detailed data).

\subsection{Interspecies quantitative metabolome comparison}

By using the optimized quenching protocol, an accurate determination of intracellular and extracellular metabolite amounts were determined in $P$. pastoris growing in aerobic carbon-limited chemostat cultures at a dilution rate of $0.1 \mathrm{~h}^{-1}$. These values were then compared with the pool sizes found in $S$. cerevisiae grown at equal culture conditions (Canelas et al. 2008). In Table 3, a summary of the rates obtained for both yeast are represented indicating higher $q_{\mathrm{CO}_{2}}, q_{\mathrm{O}_{2}}$ and $q_{\mathrm{glc}}$ rates in S. cerevisiae which lead to a lower biomass yield compared to $P$. pastoris.

\subsubsection{Intracellular metabolite pools}

When comparing the intracellular metabolite pools of $P$. pastoris and S. cerevisiae (Fig. 4), no significant differences in the profiles could be seen in upper glycolysis. Nevertheless, in the lower part of glycolysis, the 2PG/3PG and PEP pools were lower in $P$. pastoris indicating possible differences in the thermodynamic behaviour among the yeasts. The metabolite levels of the TCA cycle were similar whereby the citric acid and malate were the most abundant in both microorganisms. Also for 6PG, G3P and M6P the levels were similar. However, the T6P pool in $S$. cerevisiae was one order of magnitude larger than in $P$. pastoris. As it is known that this metabolite has an inhibitory effect on the hexokinases (Eastmond and Graham 2003) a lower glucose consumption rate could in principle be expected in S. cerevisiae if the capacity of hexokinase is similar in both organisms. However, as the maximum glucose uptake rate is higher in S. cerevisiae, i.e. $20.2 \mathrm{mmol} / \mathrm{gDCW} \cdot \mathrm{h}$ (Heyland et al. 2009), compared to P. pastoris, i.e. $2.88 \mathrm{mmol} / \mathrm{gDCW} \cdot \mathrm{h}$ (Sohn et al. 2010), it

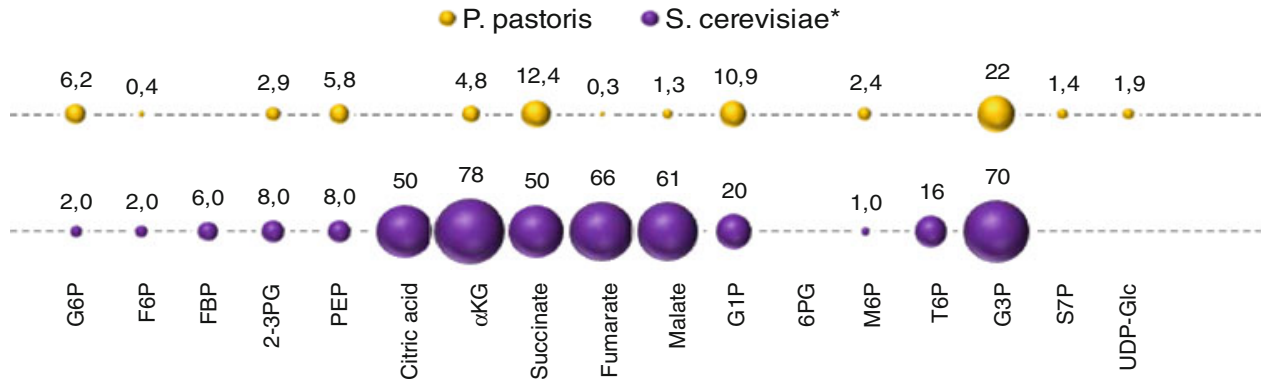

Fig. 6 Extracellular levels of central metabolites in glucose limited cultures of $P$. pastoris and $S$. cerevisiae. The bubble areas represent the percentage of the total pool which is present in the culture filtrate.
The numbers above each the bubbles represent the exact value. The metabolites without value could not be detected. *Data taken from Canelas et al. (2008) 
Fig. 7 Intracellular amino acid comparison of $P$. pastoris and $S$. cerevisiae. The bubble area represents the proportional pool sizes of each intracellular metabolite. The number above each bubble is the exact pool size in $\mu \mathrm{mol} / \mathrm{gDCW}$. *Data taken from Canelas et al. (2008)
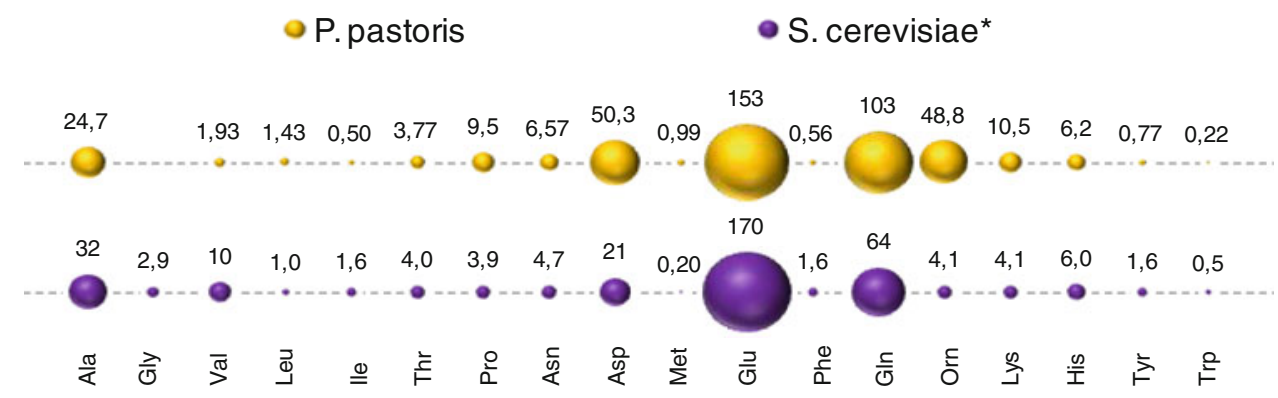

seems logical that the T6P level is higher in the former microorganism to get similar glucose consumption rates, assuming similar regulation in both yeasts.

Moreover, the mass action ratios (MAR) of enzymes from the central carbon metabolism which are expected to operate close to equilibrium (i.e. PGI, PGM, PMI, ENO and $\mathrm{FMH}$ ) were calculated (Table 4) giving more information about the thermodynamic properties of these enzymes in both yeasts. Except for enolase, no significant differences could be observed in the calculated MAR's for these enzymes for both yeasts and they were all close to the equilibrium constant. However, the calculated MAR of enolase for $P$. pastoris was significantly lower than the equilibrium constant indicating a lower capacity of enolase in $P$. pastoris compared to $S$. cerevisiae. A comparable low value of the MAR of enolase in $S$. cerevisiae was measured at a much higher growth rate of $0.33 \mathrm{~h}^{-1}$ (Canelas et al. 2011).

\subsubsection{Estimated turnover times of central metabolites}

When using the accurate determination of intracellular metabolite levels combined with the metabolic flux data from $P$. pastoris grown under analogous conditions (Baumann et al. 2010) an estimation of the turnover times for these metabolites can be calculated (Fig. 5). These turnover times are known to be an overestimation of the real turnovers inside the cell due to the usage of net fluxes instead of forward and reverse fluxes. However, even assuming that, it is interesting to see that there are already values in the order of seconds or less (FBP, PEP and 2-3PG) which highlight the importance of the rapid sampling and optimized quenching to obtain the most accurate quantification. It can be seen from Fig. 5 that the turnover times of the central metabolite pools of $P$. pastoris and $S$. cerevisiae cultivated in glucose limited chemostat under the same conditions have the same profiles, showing smaller turnover times for the intermediates of the glycolysis pathway compared to the TCA cycle.

\subsubsection{Extracellular metabolite pools}

In addition to intracellular metabolite amounts, the extracellular metabolite levels (Fig. 6) were compared for both yeasts. In this case, the $P$. pastoris culture filtrate samples were taken from the quenching experiment, hat are, under validated metabolic steady state conditions. Interestingly, in $P$. pastoris chemostat cultivations much lower extracellular metabolite levels were observed, i.e. in total $4.9 \mu \mathrm{mol} / \mathrm{gDCW}$ were found in $P$. pastoris, compared to the $41.8 \mu \mathrm{mol} / \mathrm{gDCW}$ for $S$. cerevisiae. In Fig. 6, the extracellular amounts are expressed for each metabolite as percentage of the whole broth sample amount. It can be seen from this figure that for all measurements metabolites the extracellular amounts are much lower in $P$. pastoris compared to $S$. cerevisiae and that in the latter the majority of the metabolites present outside were intermediates of TCA cycle and G3P.

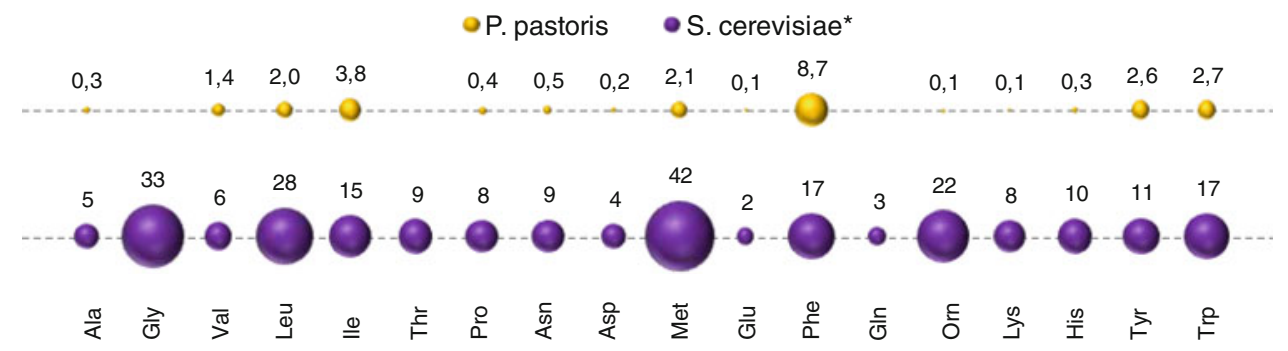

Fig. 8 Extracellular amino acid comparison of $P$. pastoris and $S$. cerevisiae. The bubble areas are proportional to \% extracellular pools of each intracellular metabolite. The numbers over the bubbles are the

exact $\%$ value. The metabolites without value could not be identified extracellularly 


\subsubsection{Intracellular amino acid pools}

The free amino acid pools measured for $P$. pastoris were compared with those previously published for $S$. cerevisiae under the same conditions (Fig. 7). Overall, amino acid pools sizes seem to follow similar trends in both microorganisms, with Glu, Ala and Asp being the major amino acids. However, in $P$. pastoris the Gln, Orn and Lys pools were larger than in $S$. cerevisiae indicating differences in the cell physiology resulting in higher accumulation levels of the amino acids derived from $\alpha \mathrm{KG}$. Moreover, Val and Met amounts were one order of magnitude higher in S. cerevisiae, even though the protein production demand of these amino acids was similar for both yeasts (based on amino acid composition of the biomass protein content taken from for $P$. pastoris (Carnicer et al. 2009) and for S. cerevisiae (Lange et al. 2001)).

In order to have a general view of the amino acid distribution, the pool sizes of all the amino acids with the same precursor were combined. Interestingly, all amino acid families were within similar range in both strains except for the glutamate family which was higher in $P$. pastoris as mentioned before which leads to a larger intracellular amino acid content in P. pastoris $(425 \mu \mathrm{mol} /$ $\mathrm{gDCW})$ compared to $S$. cerevisiae $(340 \mu \mathrm{mol} / \mathrm{gDCW})$.

\subsubsection{Extracellular amino acid pools}

In the same way as for the extracellular levels of the central metabolites, the extracellular amino acids levels were compared for both yeasts. As was found for the central metabolites, also the total level of extracellular amino acids in $P$. pastoris chemostat cultivations was much lower (total extracellular amino acid pool of $0.7 \mu \mathrm{mol} / \mathrm{gDCW}$ ) compared to $S$. cerevisiae $(13.7 \mu \mathrm{mol} / \mathrm{gDCW})$. In Fig. 8 , the extracellular amounts for each amino acid are expressed as percentage of the whole broth sample amount. It can be concluded from the low extracellular metabolite amounts in $P$. pastoris that total broth extraction would be a valid alternative for the cold filtration method because for the majority of the metabolites measured, the extracellular amounts are too low to interfere significantly with the intracellular measurement. Removal of the extracellular medium, e.g. by cold filtration or cold centrifugation) would then only be required if constituents of the medium would interfere with the analysis method applied. Furthermore, these low extracellular metabolite levels make $P$. pastoris attractive as a cell factory because a less contaminated broth facilitates the downstream processing.

\section{Conclusions}

The aim of this study was to perform a systematic investigation of a cold methanol quenching method, combined with filtration for removal of extracellular metabolites, specifically optimised for $P$. pastoris, thereby providing a validated technique for metabolomics studies of this yeast. Moreover, this methodology has been successfully applied to investigate the evolution of $P$. pastoris cells after a shift from batch to continuous operation mode under welldefined and controlled bioreactor cultivations. Notably, the obtained data has allowed performing a vis-a-vis comparison of $P$. pastoris and $S$. cerevisiae metabolomes, revealing similar profiles except for the lower glycolysis.

Overall, the outcome of this study will provide a starting point for the microbial metabolomics community future studies related to systems biology and systems metabolic engineering of $P$. pastoris.

Acknowledgments This work has been supported by the Spanish program on Chemical Process Technologies (project CTQ200760347/PPQ) of the Spanish Ministry of Science and Innovation, the Generalitat de Catalunya (Contract Grant 2009-SGR-281 and Xarxa de Referència en Biotecnologia).

Open Access This article is distributed under the terms of the Creative Commons Attribution Noncommercial License which permits any noncommercial use, distribution, and reproduction in any medium, provided the original author(s) and source are credited.

\section{Appendix}

Specific metabolite consistency index for each protocol tested. Values above 3.84 are an indicator of gross measurement errors with a confidence level of $95 \%$. Higher $h$ values are represented by bold, bold italics, italics, italics underlined and underlined fonts with increasing index values (see Table 5).

Table 5 Specific metabolite consistency index for each protocol tested

\begin{tabular}{llrrrr}
\hline Protocol & \multicolumn{7}{l}{$h$-Index } & & & \\
\cline { 2 - 6 } & $\mathrm{A}$ & $\mathrm{B}$ & $\mathrm{C}$ & $\mathrm{D}$ & $\mathrm{E}$ \\
\hline G6P & 8.2 & $\underline{1.5}$ & 7.7 & $\underline{4.1}$ & $\underline{1.6}$ \\
F6P & $\underline{4.4}$ & 0.8 & 0.1 & 0.0 & $\underline{1.8}$ \\
2-3PG & $\underline{3.4}$ & 0.1 & $\underline{5.4}$ & 0.0 & 0.2 \\
PEP & $\underline{1.5}$ & $\underline{5.4}$ & $\underline{1.4}$ & $\underline{2.7}$ & $\underline{\mathbf{3 . 1}}$ \\
Pyruvate & $\underline{7.8}$ & $\mathbf{2 5 . 8}$ & $\mathbf{1 6 . 3}$ & $\mathbf{1 2 . 6}$ & $\underline{9.0}$ \\
$\alpha$ KG & $\underline{2.8}$ & 0.0 & $\mathbf{1 1 . 1}$ & 0.0 & $\underline{2.9}$ \\
Succinate & 0.2 & $\underline{4.6}$ & 0.2 & 0.8 & $\underline{1.9}$ \\
Fumarate & 9.7 & $\underline{6.4}$ & $\underline{1.9}$ & 0.6 & $\underline{1.0}$ \\
Malate & 0.1 & 0.1 & 0.1 & 0.1 & 0.2 \\
G1P & $\underline{2.8}$ & $\underline{1.9}$ & $\underline{1.5}$ & $\underline{0.8}$ & $\mathbf{2 3 . 0}$ \\
6PG & 0.4 & 0.6 & 0.0 & 0.0 & 0.2 \\
M6P & $\underline{3.2}$ & $\underline{1.4}$ & $\underline{3.9}$ & 8.7 & $\underline{4.7}$ \\
\hline
\end{tabular}


Table 5 continued

\begin{tabular}{llllll}
\hline Protocol & $h$-Index & & & & \\
\cline { 2 - 6 } & $\mathrm{A}$ & $\mathrm{B}$ & $\mathrm{C}$ & $\mathrm{D}$ & $\mathrm{E}$ \\
\hline G3P & 0.4 & 0.3 & $\underline{4.2}$ & $\mathbf{1 1 . 2}$ & 0.0 \\
S7P & 0.0 & 0.2 & 0.8 & $\underline{1.7}$ & 0.0 \\
UDP-glc & 0.7 & 0.4 & 0.0 & 0.0 & 0.3 \\
Ala & 0.5 & $\underline{1.3}$ & 0.0 & 0.0 & $\underline{1.6}$ \\
Gly & 9.0 & $\underline{6.5}$ & $\mathbf{1 4 . 2}$ & $\underline{5.4}$ & $\underline{4.0}$ \\
Val & $\underline{2.9}$ & $\underline{4.0}$ & 0.0 & 0.8 & $\underline{2.3}$ \\
Leu & $\underline{2.1}$ & $\underline{1.4}$ & 0.0 & 5.8 & $\underline{2.7}$ \\
Ile & $\underline{3.3}$ & $\underline{2.1}$ & 0.6 & $\underline{3.6}$ & $\underline{3.3}$ \\
Thr & 0.1 & 0.8 & 0.2 & 0.4 & 0.2 \\
Pro & 0.0 & 0.1 & $\underline{1.1}$ & $\underline{2.8}$ & 0.1 \\
Asn & 0.1 & 0.1 & 0.9 & $\underline{2.1}$ & 0.1 \\
Asp & $\underline{2.2}$ & 0.6 & $\underline{2.1}$ & $\underline{3.1}$ & 0.0 \\
Met & 0.8 & 0.0 & 0.0 & $\underline{2.4}$ & 0.2 \\
Glu & 0.0 & 0.2 & 0.6 & $\underline{2.0}$ & 0.0 \\
Phe & $\underline{2.1}$ & $\underline{3.0}$ & 7.0 & $\mathbf{2 4 . 0}$ & 8.5 \\
Gln & 0.0 & 0.0 & $\underline{1.1}$ & $\underline{1.2}$ & 0.1 \\
Orn & 0.0 & 0.2 & 0.3 & $\underline{1.6}$ & 0.4 \\
Lys & 0.0 & 0.0 & 0.4 & $\underline{1.4}$ & 0.1 \\
His & 0.2 & 0.4 & 0.2 & $\underline{1.2}$ & $\underline{1.1}$ \\
Tyr & $\underline{1.3}$ & 0.3 & 0.0 & 0.1 & $\underline{1.7}$ \\
Trp & $\underline{3.7}$ & 0.1 & 0.1 & $\underline{1.5}$ & $\underline{1.4}$ \\
\hline & & & & &
\end{tabular}

\section{References}

Abbott, B. J., Laskin, A. I., \& McCoy, C. J. (1974). Effect of growth rate and nutrient limitation on the composition and biomass yield of Acinetobacter calcoaceticus. Applied Microbiology, 28, $58-63$.

Baumann, K., Maurer, M., Dragosits, M., Cos, O., Ferrer, P., \& Mattanovich, D. (2008). Hypoxic fed-batch cultivation of Pichia pastoris increases specific and volumetric productivity of recombinant proteins. Biotechnology and Bioengineering, 100, 177-183.

Baumann, K., et al. (2010). A multi-level study of recombinant Pichia pastoris in different oxygen conditions. BMC Systems Biology, $4,141$.

Bollok, M., Resina, D., Valero, F., \& Ferrer, P. (2009). Recent patents on the Pichia pastoris expression system: Expanding the toolbox for recombinant protein production. Recent Patents on Biotechnology, 3, 192-201.

Bolten, C. J., Kiefer, P., Letisse, F., Portais, J.-C., \& Wittmann, C. (2007). Sampling for metabolome analysis of microorganisms. Analytical Chemistry, 79, 3843-3849.

Bolten, C. J., \& Wittmann, C. (2008). Appropriate sampling for intracellular amino acid analysis in five phylogenetically different yeasts. Biotechnology Letters, 30, 1993-2000.

Canelas, A. B., Ras, C., ten Pierick, A., van Dam, J. C., Heijnen, J. J., \& van Gulik, W. M. (2008). Leakage-free rapid quenching technique for yeast metabolomics. Metabolomics, 4, 226-239.

Canelas, A. B., Ras, C., ten Pierick, A., van Gulik, W. M., \& Heijnen, J. J. (2011). An in vivo data-driven framework for classification and quantification of enzyme kinetics and determination of apparent thermodynamic data. Metabolic Engineering. doi: 10.1016/j.ymben.2011.02.005.

Canelas, A. B., ten Pierick, A., Ras, C., Seifar, R. M., van Dam, J. C., van Gulik, W. M., et al. (2009). Quantitative evaluation of intracellular metabolite extraction techniques for yeast metabolomics. Analytical Chemistry, 81, 7379-7389.

Carnicer, M., Baumann, K., Töplitz, I., Sánchez-Ferrando, F., Mattanovich, D., Ferrer, P., et al. (2009). Macromolecular and elemental composition analysis and extracellular metabolite balances of Pichia pastoris growing at different oxygen levels. Microbial Cell Factories, 8, 65.

Cascante, M., \& Marin, S. (2008). Metabolomics and fluxomics approaches. In Essays in biochemistry (pp. 67-82). London: Portland Press.

Cereghino, G. P. L., Cereghino, J. L., Ilgen, C., \& Cregg, J. M. (2002). Production of recombinant proteins in fermenter cultures of the yeast Pichia pastoris. Current Opinion in Biotechnology, 4, 329-332.

Chung, B. K. S., Selvarasu, S., Camattari, A., Ryu, J., Lee, H., Ahn, J., et al. (2010). Genome-scale metabolic reconstruction and in silico analysis of methylotrophic yeast Pichia pastoris for strain improvement. Microbial Cell Factories, 9, 50-64.

Cos, O., Ramón, R., Montesinos, J. L., \& Valero, F. (2006). Operational strategies, monitoring and control of heterologous protein production in the methylotrophic yeast Pichia pastoris under different promoters: A review. Microbial Cell Factories, $5,17$.

Dettmer, K., Aronov, P. A., \& Hammock, B. D. (2007). Mass spectrometry-based metabolomics. Mass Spectrometry Reviews, 26, 51-78

Douma, R. D., Jonge, L. P., de Jonker, C. T. H., Seifar, R. M., Heijnen, J. J., \& van Gulik, W. M. (2010a). Intracellular metabolite determination in the presence of extracellular abundance: Application to the penicillin biosynthesis pathway in Penicillium chrysogenum. Biotechnology and Bioengineering, 107, 105-115.

Douma, R. D., Verheijen, P. J. T., Laat, W. T., de Heijnen, J. J., \& van Gulik, W. M. (2010b). Dynamic gene expression regulation model for growth and penicillin production in Penicillium chrysogenum. Biotechnology and Bioengineering, 106, 608-618.

Eastmond, P., \& Graham, I. A. (2003). Trehalose metabolism: A regulatory role for trehalose-6-phosphate? Current Opinion in Plant Biology, 6, 231-235.

Heyland, J., Fu, J., \& Blank, L. M. (2009). Correlation between TCA cycle flux and glucose uptake rate during respiro-fermentative growth of Saccharomyces cerevisiae. Microbiology, 155, 3827-3837.

Heyland, J., Fu, J., Blank, L. M., \& Schmid, A. (2010). Quantitative physiology of Pichia pastoris during glucose-limited high-cell density fed-batch cultivation for recombinant protein production. Biotechnology and Bioengineering, 107, 357-368.

Lange, H. C., Eman, M., Zuijlen, G., van Visser, D., van Dam, J. C., Frank, J., et al. (2001). Improved rapid sampling for in vivo kinetics of intracellular metabolites in Saccharomyces cerevisiae. Biotechnology and Bioengineering, 75, 406-415.

Macauley-Patrick, S., Fazenda, M. L., McNeil, B., \& Harvey, L. M. (2005). Heterologous protein production using the Pichia pastoris expression system. Yeast, 22, 249-270.

Mashego, M. R., Wu, L., van Dam, J. C., Ras, C., Vinke, J. L., van Winden, W. A., et al. (2004). MIRACLE: Mass isotopomer ratio analysis of $\mathrm{U}_{-}{ }^{13} \mathrm{C}$-labeled extracts. A new method for accurate quantification of changes in concentrations of intracellular metabolites. Biotechnology and Bioengineering, 85, 620-628.

Nielsen, J. (1997). Metabolic control analysis of biochemical pathways based on a thermokinetic description of reaction rates. Biochemistry Journal, 321, 133-138. 
Oldiges, M., Lütz, S., Pflug, S., Schroer, K., Stein, N., \& Wiendahl, C. (2007). Metabolomics: Current state and evolving methodologies and tools. Applied Microbiology and Biotechnology, 76, 495-511.

Pscheidt, B., \& Glieder, A. (2008). Yeast cell factories for fine chemical and API production. Microbial Cell Factories, 7, 25.

Sohn, S. B., Graf, A. B., Kim, T. Y., Gasser, B., Maurer, M., Ferrer, P., et al. (2010). Genome-scale metabolic model of methylotrophic yeast Pichia pastoris and its use for in silico analysis of heterologous protein production. Biotechnology Journal, 5, 705-715.

Solà, A., Jouhten, P., Maaheimo, H., Sánchez-Ferrando, F., Szyperski, T., \& Ferrer, P. (2007). Metabolic flux profiling of Pichia pastoris grown on glycerol/methanol mixtures in chemostat cultures at low and high dilution rates. Microbiology, 153, 281-290.

Solà, A., Maaheimo, H., Ylönen, K., Ferrer, P., \& Szyperski, T. (2004). Amino acid biosynthesis and metabolic flux profiling of Pichia pastoris. European Journal of Biochemistry, 271, 2462-2470.

Tang, Y. J., Garcia Martin, H., Myers, S., Rodriguez, S., Baidoo, E. E. K., \& Keasling, J. D. (2009). Advances in analysis of microbial metabolic fluxes via ${ }^{13} \mathrm{C}$ isotopic labeling. Mass Spectrometry Reviews, 28, 362-375.

Taymaz-Nikerel, H., Mey, M., de Ras, C., ten Pierick, A., Seifar, R. M., van Dam, J. C., et al. (2009). Development and application of a differential method for reliable metabolome analysis in Escherichia coli. Analytical Biochemistry, 386, 9-19.

Tredwell, G. D., Edwards-Jones, B., Leak, D. J., \& Bundy, J. G. (2011). The development of metabolomic sampling procedures for Pichia pastoris, and baseline metabolome data. PLOS ONE, 6, 1-16. e16286. van Dam, J. C., Eman, M., Frank, J., Lange, H. C., van Dedem, G. W. K., \& Heijnen, J. J. (2002). Analysis of glycolytic intermediates in Saccharomyces cerevisiae using anion exchange chromatography and electrospray ionization with tandem mass spectrometric detection. Analytica Chimica Acta, 460, 209-218.

van der Heijden, R. T., Romein, B., Heijnen, J. J., Hellinga, C., \& Luyben, K. C. (1994). Linear constraint relations in biochemical reaction systems: II. Diagnosis and estimation of gross errors. Biotechnology and Bioengineering, 43, 11-20.

van Gulik, W. M. (2010). Fast sampling for quantitative microbial metabolomics. Current Opinion in Biotechnology, 21, 27-34.

Verheijen, P. J. T. (2010). Data reconciliation and error detection. In C. D. Smolke (Ed.), The metabolic pathway engineering handbook (pp. 8.1-8.13). Boca Raton: CRC Press.

Villas-Bôas, S. G., Højer-Pedersen, J., Akesson, M., Smedsgaard, J., \& Nielsen, J. (2005). Global metabolite analysis of yeast: Evaluation of sample preparation methods. Yeast, 22, $1155-1169$.

Wellerdiek, M., Winterhoff, D., Reule, W., Brandner, J., \& Oldiges, M. (2009). Metabolic quenching of Corynebacterium glutamicum: Efficiency of methods and impact of cold shock. Bioprocess and Biosystems Engineering, 32, 581-592.

Wittmann, C., Krömer, J. O., Kiefer, P., Binz, T., \& Heinzle, E. (2004). Impact of the cold shock phenomenon on quantification of intracellular metabolites in bacteria. Analytical Biochemistry, 327, 135-139.

Wu, L., Mashego, M. R., van Dam, J. C., Proell, A. M., Vinke, J. L., Ras, C., et al. (2005). Quantitative analysis of the microbial metabolome by isotope dilution mass spectrometry using uniformly ${ }^{13} \mathrm{C}$-labeled cell extracts as internal standards. Analytical Biochemistry, 336, 164-171. 